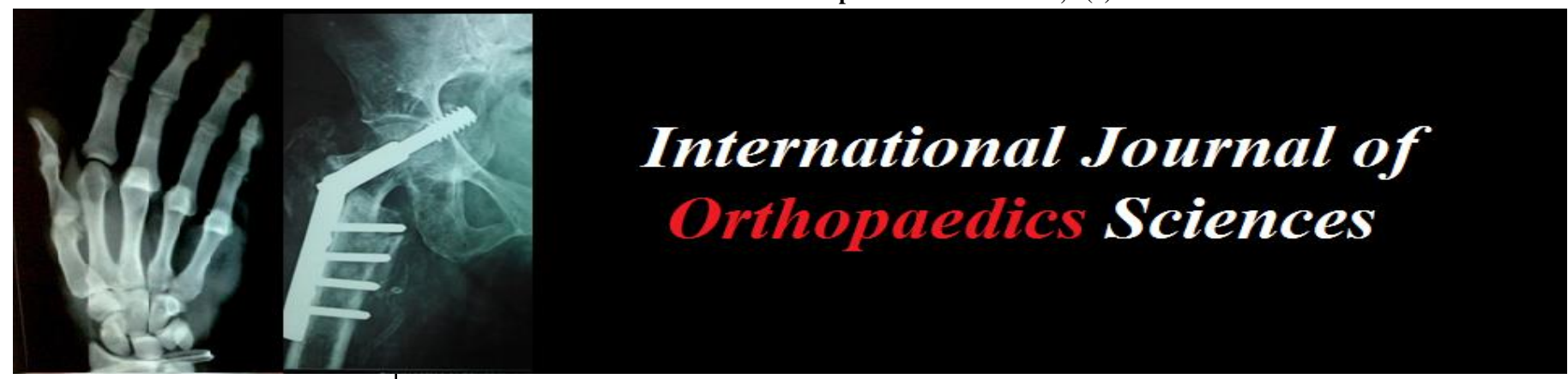

E-ISSN: 2395-1958

P-ISSN: 2706-6630

IJOS 2019; 5(4): 393-398

(C) 2019 IJOS

www.orthopaper.com

Received: 20-08-2019

Accepted: 24-09-2019

\section{Dr. Shivanna}

Assistant Professor, Department of

Orthopaedics, Chamarajanaga

Institute of Medical Sciences

Chamarajanagar, Karnataka, India

\section{Dr. Krishnaprasad}

Senior Resident and RMO,

Department of orthopaedics,

Chamarajanagar Institute of

Medical Sciences Chamarajanagar,

Karnataka, India
Corresponding Author:

Dr. Shivanna

Assistant Professor, Department of orthopaedics, Chamarajanagar Institute of Medical Sciences Chamarajanagar, Karnataka, India

\title{
Correction of neglected clubfoot in adults: Our results
}

\section{Dr. Shivanna and Dr. Krishnaprasad}

DOI: https://doi.org/10.22271/ortho.2019.v5.i4g.1704

\section{Abstract}

Summary: The neglected clubfoot deformity is a major orthopaedic disabler of children and adults in developing nations. The bones and joints of the foot deform into fixed equinus, adductus, cavus, and supination and as patients walk on the side or dorsum of the foot, the deformity is exaggerates. This severe increasing disability physically and mentally can be significantly become better with surgical correction.

Objective: This study aimed to evaluate the triple arthrodesis and posterior tendoachilis lengthening for rigid neglected clubfoot deformities in adult patients, and its effects on clinical and radiological results.

Methods: The procedures were performed in one session on 16 feet of 12 patients [ 7 male, 5 female; mean age 21 (range: 15 to 30)]. $\mathrm{K}$ wires were used for fixation in all patients. The AOFAS (American Orthopaedic Foot and Ankle Society) ankle- hindfoot scale was used for clinical assessment.

The mean follow-up was 4 years (range: 1.5 to 6 ).

Results: The mean AOFAS score rose from a preoperative 36 (range: 26 to 52) to 90 (range: 86 to 94) in the final follow up ( $p<0.0001)$. Among the 16 feet, 12 were evaluated as excellent, 4 as good. Significant clinical improvement was obtained between preoperative and postoperative surgical periods $(p<0.05)$. Significant improvements were observed in radiographic parameters $(p<0.0001)$.

At final follow-up, radiographic values were within normal physiological limits. Average union time was 7 weeks (range: 5 to 10). Pseudoarthrosis, delayed union and talus avascular necrosis was not observed in any patients.

Conclusion: The good surgical technique of triple arthrodesis and Tendo Achilis lengthening yields better results cosmetically and functionally in neglected clubfoot cases with severe deformities.

Keywords: Neglected clubfoot. triple arthrodesis, Tendoachilis

\section{Introduction}

The neglected clubfoot is a disabling orthopaedic deformity in the developing countries. Approximately $80 \%$ of children born with a clubfoot deformity are born in the developing world, and the large majority of these do not have access to appropriate medical care. The obstacles of poverty, lack of awareness, and lack of appropriate medical resources in accessible locations that treatment is either not initiated or incompletely performed. The orthopaedic literature on clubfoot deformity focuses on early intervention in a resource-rich environment, with numerous surgical options outlined for both primary treatment and treatment of the relapsed clubfoot ${ }^{[1,2]}$. There is very little literature available on treatment of the neglected clubfoot with major texts providing little more than anecdotal reference to triple arthrodesis as a salvage.

The neglected clubfoot, however, is one in which there has been no initial treatment or perhaps very inadequate and incomplete initial treatment. The deformity is made worse at the time the child starts to walk because weight bearing takes place on the side or dorsum of the foot, exaggerating the abnormal shape and causing further deformation. The contracted soft tissues on the medial side of the foot are encouraged to contract further. The bones are compressed unnaturally at a time when they are plastic and deform into abnormal shapes. Bones that normally support the arch of the midfoot now bear axial load, which they were never intended to do. The sole of the foot never experiences proper weight bearing, and it is impossible to wear normal shoes. 
A thickened callous and large bursa develop over the prominent weight bearing head of the talus on the dorso lateral side of the foot, often associated with deep fissures, which are vulnerable to breakdown and infection.

While relations of the deformed ankle, tarsal and metatarsal bones are not symptomatic in the early period, they cause degeneration and resultant pain between bones in the later period ${ }^{[3,4]}$. Unless treated prior to completion of skeletal maturity, this deformity becomes more severe, as in the cases presented here ${ }^{[4]}$

Conservative methods are very effective in the treatment of clubfoot deformity in children, with surgical treatment only preferred in cases that do not respond to conservative treatment or are neglected. In early childhood, soft tissue procedures (capsulotomies, tendon lengthening, tendon transfers etc.) are common, while soft tissue releases alone do not treat deformities in adults, among whom the recurrence rate is high. Therefore, in adults, soft tissue procedures are carried out together with bone operations (several osteotomies and arthrodesis etc.) or combined soft tissue release ${ }^{[5,3,6]}$. Today, triple arthrodesis has become common in the treatment of neglected clubfoot deformity [3] Triple arthrodesis is the combined fusion of the talonavicular, calcaneocuboid and subtalar joints ${ }^{[6]}$. It is asafe and reliable method, used in traumatic, neurological and rheumatic disorders of the foot $[3,4,7]$. It aims to eliminate deforming forces, stop deformity development and, by minimizing or totally stopping device-dependent life, rearrange the foot in terms of stability and statics, thus making it possible to obtain a whole cosmetically, functionally and anatomically nearnormal foot ${ }^{[8-10]}$.

\section{Pathologic anatomy}

The primary contractile forces of the soft tissues in clubfoot deformity result in progressive bony deformity. These primary soft tissue and bony deformities have been well described in the dissections of Ponseti 11 and in the magnetic resonance imaging studies of Pirani.12 The neck of the talus develops a medial angulation and the head of the talus becomes conical in shape. The navicular bone articulates with the medial aspect of the head of the talus and becomes wedgeshaped. The calcaneocuboid joint becomes oblique with medial subluxation of the cuboid on the calcaneus. The subtalar joint is held in its maximal position of supination (plantar flexion, inversion, and adduction). Early manipulation and cast treatment using the Ponseti technique shows cartilage remodelling back toward a normal anatomic appearance.12 If the deformity persists as a neglected clubfoot, bony deformity becomes more entrenched because there is progressively less ability to remodel. Therefore, the neglected clubfoot shows all of the neonatal elements reflected in the osseous structure. There is significant bony deformity in the neck and head of the talus, shape of the navicular, subluxation of the cuboid, and dramatic obliquity of the calcaneocuboid joint. Soft tissue release alone cannot fully restore bony anatomy, and the relapse rate in the older child with a neglected clubfoot will be high because of the tendency of the bones to revert to their deformed position. Of particular importance is the obliquity of the calcaneo-cuboid joint, and surgical procedures on the neglected clubfoot must address this bony incongruency as a primary consideration.

The degree of fixed cavus will determine how the child walks. If the cavus is not severe, or is flexible, the child will tend to walk on the lateral border of the foot with the forefoot still facing forward. With large degrees of fixed cavus deformity, the foot may face backward (Fig. 1). In both clinical scenarios, the degree of equinus of the rear foot is not readily apparent when standing but becomes obvious when the forefoot adductus is corrected. Dramatic and fixed equinus remains the most problematic of the clubfoot deformities to correct at all ages and degrees of neglected deformity.

\section{The disability of neglected clubfoot deformity}

Children with a neglected clubfoot deformity do learn to walk without the use of crutches or walking aids. They can even run over short distances. Qualitative research in previous studies indicated that the neglected clubfoot deformity was indeed a significant disability for children, preventing access to education and other social activity.13 The children are often considered cursed or unworthy of advancement in education or social status.

There is pain and difficulty with locomotion is experienced over longer distances. There is also abnormal pressure distribution across the midtarsal joints and through the malaligned ankle joint causing pain and early degenerative changes in foot and ankle. Recurrent skin breakdown with infections is not uncommon in the skin bearing weight on the dorsal and lateral aspect of the foot. Severe ulceration in adults can lead to chronic osteomyelitis and amputation. There is an inability to wear footwear, which aggravates all of the previously mentioned problems. The aim of obtaining a plantigrade foot that can fit normal shoes is worthwhile even if feet do not have normal mobility or shape and size, or have some degree of residual pain. Success of treatment of the neglected clubfoot can be evaluated by two primary indicators: weight bearing on the skin of the sole of the foot and the ability to wear normal shoes. Yadav has reported $87 \%$ acceptable results using these criteria. 14

\section{The technique of triple arthrodesis}

A standard oblique Ollier-type incision is made on the dorso lateral side of the foot. The lateral incision starting at the cuboid bone extending to talo navicular joint, soft tissue exposed. The bursa excised in toto. The extensor brevis then sharply dissected and retracted distally. The extensor digitorum then protected and retracted medially. Calcaneo cuboid joint to talo navicular joint soft tissue dissected and exposed. A dorso lateral based wedge then marked from calcaneo cuboid joint with the angle forming at talo navicular joint. The angle marked should correspond to the angle of adduction and residual cavus deformity. Special care to be taken to excise the cartilaginous tissue if any in order to prevent pseudoarthrosis or non union of osteotomy.

With the sharp dissection through the same incion proximally subtalar joint exposed. Lateral based wedge osteotomy done. The correction is accomplished by depressing the neck of the talus into the notch on the undersurface of the navicular, subluxating the foot dorsally while abducting the forefoot. An additional small amount of equinus correction can be achieved by percutaneous or open lengthening of the Achilles tendon. Plantar fasciotomy can also be added for cavus, performed through a short longitudinal incision in the midfoot.

Internal fixation of osteotomy surfaces is preferable. In smaller feet, there is not enough space for staples, and K-wire fixation, therefore, becomes the standard. In larger feet, staples may be used. In a follow-up study of triple arthrodesis in children performed in Uganda, there was a pseudoarthrosis rate of $33 \%$ at the talonavicular joint. 15 Fixation was not used and likely contributed to this high rate. The small size of the 
deformed navicular in neglected clubfoot cases and the subluxation dorsally created by the Lambrinudi triple arthrodeses gives very small surface contact for arthrodesis.

This same study showed approximately two thirds of patients has some degree of residual deformity, as well as some degree of chronic pain in 38\%15. A high proportion (92\%) of patients, however, was happy with the procedure because it gave them a plantigrade foot that could use shoes. In a similar follow-up study of triple arthrodesis for neglected clubfoot in 64 patients over 10 years of age, Hersh and Fuchs found 89\% of patients happy with the procedure ${ }^{[16]}$.

Herold and Torok described a two-stage correction for neglected clubfeet in older children and adults ${ }^{[17]}$ The first stage consisted of extensive posteromedial release, followed by serial casting at two weekly intervals. Several weeks later, a second stage bony procedure was performed, consisting of tarsal wedge osteotomy or triple arthrodeses. Their strategy was to minimize bone resection by preliminary soft tissue correction and serial casting. Their procedure obviously used more surgical resources and required more time commitment than a single-stage procedure. Yadav in India likewise performed triple arthrodeses in association with extensive soft tissue release and reported good results maintained over time.14 $\mathrm{He}$ did not report on the incidence of avascular necrosis of the talus. Avascular necrosis is a known complication of triple arthrodeses surgery ${ }^{[5]}$. Resection of bone through a lateral incision and cleaning out the sinus tarsi eliminates the lateral side vascular supply to the head of the talus. It would seem prudent, therefore, to avoid any disruption of the important medial blood supply coming in through the deltoid ligament and tarsal canal. This study analyzed the results of triple arthrodesis and tendoachilis lengthening performed on adult patients with neglected clubfoot for which soft tissue surgery alone was insufficient. The aim of the study was to show that correcting surgically of these deformities will significantly improve cosmetic appearance and physical performance of these patients which make them more confident in their life.

\section{Patients and methods}

The study was carried out retrospectively with data prospectively collected between 2014 and 2019. sixteen feet of 12 patients ( 7 male, 5 female) who underwent triple arthrodesis and tendoachilis lengthening were followed and checked. The patients had extremely rigid foot structures that could not be corrected through passive manipulation (Fig. 1). Of the patients, 4 had bilateral deformity. All 4 had both feet operated. Patient mean age was 21 (range: 15 to 30 ).

Eight patients had left side involvement, and 8 right side. The mean follow-up was 4years (range: 1.5 to 6) (Table 1). None of the patients had undergone any previous surgical or nonsurgical treatment.

Table 1: Patient demographics and AOFAS degrees.

\begin{tabular}{|c|c|c|c|c|c|c|}
\hline \multirow{2}{*}{ No } & \multirow{2}{*}{ gender } & \multirow{2}{*}{ age } & \multirow{2}{*}{ Side } & \multirow{2}{*}{ Union time (weeks) } & \multicolumn{2}{|c|}{ AOFAS Ankle-Hind Foot Scale } \\
\cline { 5 - 7 } & & & & Pre-operative & Post-operative \\
\hline 1 & female & 26 & Right & 8.5 & 28 & 90 \\
\hline & & & Left & 6 & 32 & 88 \\
\hline 2 & male & 16 & Right & 7 & 38 & 92 \\
\hline & & & Left & 6.5 & 44 & 92 \\
\hline 3 & male & 15 & Right & 5 & 26 & 90 \\
\hline 4 & male & 18 & Right & 7 & 52 & 93 \\
\hline 5 & female & 28 & Left & 8 & 38 & 90 \\
\hline & & & Right & 7.5 & 40 & 88 \\
\hline 6 & male & 22 & Left & 8 & 28 & 90 \\
\hline 7 & male & 16 & Left & 6 & 30 & 92 \\
\hline 8 & female & 29 & Right & 8.5 & 41 & 88 \\
\hline 9 & male & 30 & Left & 10 & 26 & 86 \\
\hline 10 & female & 16 & Right & 6 & 32 & 92 \\
\hline & & & Left & 6 & 36 & 90 \\
\hline 11 & female & 20 & Right & 7 & 46 & 94 \\
\hline 12 & male & 25 & Left & 6 & 40 & 92 \\
\hline
\end{tabular}

Before operation, over-knee tourniquets were placed on all feet. First the cavus deformity was corrected with percutaneous plantar fasciotomy. Through a lateral incision starting at the cuboid bone extending to talo navicular joint, soft tissue exposed. The bursa excised in toto. The extensor brevis then sharply dissected and retracted distally. The extensor digitorum then protected and retracted medially. Calcaneo cuboid joint to talo navicular joint soft tissue dissected and exposed. A dorso lateral based wedge then marked from calcaneo cuboid joint with the angle forming at talo navicular joint. The angle marked should correspond to the angle of adduction and residual cavus deformity. Special care to be taken to excise the cartilaginous tissue if any in order to prevent pseudoarthrosis or non union of osteotomy. With the sharp dissection through the same incion proximally subtalar joint exposed. Lateral based wedge osteotomy done. Overcorrection should be avoided at this point. Multiple K wires are used to fix the osteotomies.

Tendo achilis can be lengthened either by open or percutaneous triple tenotomy. Wound are closed with suction drain.

All patients were casted in long leg circular plaster for 6 weeks and walking plaster cast for another 4 weeks. K wires were removed after 6 weeks. No braces were used after removal of plaster cast. Xrays were taken every month. Clinical and radiological examinations of the patients were carried out regularly. Monthly examinations were made in the first three months, quarterly examinations in the following one year, and subsequently six-monthly. Patients were evaluated clinically, radiologically and cosmetically. For clinical assessment, the AOFAS ankle-hindfoot scale was used to evaluate pain, function and alignment ${ }^{[18]}$.

The whole foot was examined and palpated thoroughly and united joints were determined. In the final controls, varus, valgus and neutral positions of feet were evaluated.

Functionally, a soft moving ankle, no pain after long exercise and ability to wear a shoe easily was evaluated as a good result; pain after standing for a long time and a mild 
toughness in the ankle was fair, and apparent pain after walking, inability to wear shoes easily and a tough ankle was evaluated as a bad result ${ }^{[19]}$. The patients were asked whether they were satisfied with the operation or not.

Ankle (tibiotalar joint) maximum dorsiflexion and plantar flexion movements were measured with a standard goniometer with the patient in a sitting position. Tibial long axis and the lateral side of the foot were based as references.

The period and formation of the union was determined by clinical and radiological evaluation. Criteria such as no observation of pathological movement in the foot palpation, no feeling of pain when weight was put on the foot or while wearing shoes, and no edema or trophic changes in the foot were considered as clinical parameters. Radiological parameters were based on no detection of osteotomy lines and no pseudoarthrosis-related findings.

Cosmetically, stepping plantigrade with no varus at hindfoot was evaluated as a good result, with mild varus as a fair, and inability to step plantigrade with apparent varus as a bad result ${ }^{[19]}$. In final controls, the feet's varus, valgus and neutral positions were evaluated clinically at stepping position.

Difference in foot length were noted in all patients operated on single side. Comparative measurements of the two feet were taken with a ruler while sitting on the examination couch.

Radiologically, standard anteropsterior/lateral and oblique graphs and standing anteroposterior/lateral and oblique graphs were taken. Valgus-varus measurements were made on these graphs.

\section{Results}

Mean tourniquet time was 70 (range: 60 to 90), and mean operation time was 65 minutes (range: 45 to 90). None of the patients developed subcutaneous or deep infection. No vascular complications were faced. None of the patients developed pseudoarthrosis or avascular necrosis of talus.

The mean union time was 7 (range: 5 to 10) weeks. Mean plantar flexion, which was $18^{\circ}$ (range: $10^{\circ}$ to $23^{\circ}$ ) in the preoperative period, increased in the postoperative period to $30^{\circ}$ (range: $21^{\circ}$ to $\left.35^{\circ}\right)(p<0.0001)$. Mean dorsiflexion, on the other hand, was $4^{\circ}$ (range: $1^{\circ}$ to $7^{\circ}$ ) in the preoperative period and increased to $12^{\circ}$ (range: $6^{\circ}$ to $18^{\circ}$ ) postoperatively ( $p<$ $0.0001)$. The preoperative foot length mean of $24.5 \mathrm{~cm}$ (range: 21 to $26 \mathrm{~cm}$ ) decreased to $23.7 \mathrm{~cm}$ (range: 20.4 to 25 $\mathrm{cm}$ ) postoperatively. The rate of foot shortening was found to be $0.8 \mathrm{~cm}$ (range: 0.5 to $1 \mathrm{~cm}$ ) on average. In the preoperative period, the AOFAS hindfoot stability score in all patients was 0 , meaning all were unstable. In final examinations, however, the same score was $8(p<0.05)$.

In this study of 16 feet of 12 patients, the preoperative AOFAS score was 36(range: 26 to 52) and final examination score was found to be 90 (range: 86 to 94$)(p<0.0001)$. Considering the AOFAS scale scores in combination with the functional and cosmetic results ${ }^{[12]}$, it was concluded that among the 16 feet, 12 (75\%) were excellent, 4 (25\%) were good. All the patients expressed satisfaction with the operation as they were pain-free and could step plantigrade better cosmetically.

\section{Discussion}

Combining posteromedial soft tissue releases with wedge resection osteotomies carried out at various levels on the lateral column in neglected clubfoot has been suggested by many authors ${ }^{[20-23]}$. As joint surfaces are destroyed in this method, growth is distorted and the foot remains relatively small. Some authors, on the other hand $[19,23-25]$ state that applying triple arthrodesis at a young age such as $8,{ }^{[25]}$ or at any age when the ossified centre of navicula occurs, is a saving interruption. Some authors, such as el-Tayeby, have carried out cuboid and or lateral closed calcaneal osteotomy together with massive medial soft tissue release on adolescent patients (aged 4-14). ${ }^{[26]}$ Although growth cartilages are not damaged in this method, one foot remains slightly smaller than the other. Some authors ${ }^{[6,19,27]}$ have carried out triple arthrodesis together with two-stage massive medial soft tissue release on mature feet. For 30 years, Dobbs et al. ${ }^{[28]}$ followed the 73 feet of 45 patients on whom they had performed only soft tissue procedures. Their results show that the size of soft tissue interruption is parallel with the amount of functional deformity, and that recurrent soft tissue releases cause a tough, painful and arthritic foot and decrease life quality significantly. In the long term follow-ups, small or big joint degenerations of various levels were found in the tibiotalar and tarsal joints in almost all series; pseudoarthrosis and avascular necrosis were observed, and in some, talar flattening and skin ulcers developed ${ }^{[25]}$. As is clear then, no matter the age group or method used, in almost all cases, the foot is more or less affected and complications are possible.

Slightly modifying the previous methods of triple arthrodesis and tendoachilis lengthening in mature feet for saving purposes in patients for whom no treatment alternative existed, other than triple arthrodesis. It has been stated that massive soft tissue releases on the medial decrease the amount of bones to be resected from the talus neck and subtalar joint, which decreases pseudoarthrosis, talar flattening and vascular necrosis incidence as it protects vascular structures ${ }^{[29]}$. Angus and Cowell ${ }^{[25]}$ obtained good results in 3, fair results in 6 and bad results in 8 of the 17 idiopathic clubfoot patients whom they treated without soft tissue release. They explained that bone osteotomies alone did not yield good results, especially in cases with severe equines deformities. On the other hand, Penny ${ }^{[4]}$ stated that when extensile medial release is carried out with triple arthrodesis, it carries a potential risk of devascularisation for the talus. According to Penny, it could be applied in young children, while direct triple arthrodesis is required in adults. The same study also suggested that a more proper and faster application is possible when only triple arthrodesis is carried out, and found that there was no difference between the two methods in terms of joint thickness, growth arrest and foot size.

There are several approaches to internal fixation in triple arthrodesis operations. The aim here is to decrease movement in the fusion area, maintain the continuity of reduction, and prevent recurrence and pseudoarthrosis ${ }^{[8]}$. While some authorities use no fixation materials $[19,30,31]$. others use Kwires, staples and screws ${ }^{[4,7,19,25,27]}$. However, those who do not use internal fixation materials apply a plaster cast for an average period of as long as three months. Penny [4] recommended fixation with $\mathrm{K}$-wires for small feet as there is not adequate space, and $U$ staples for bigger feet. Although many authors claim that screws are more effective than staples in rigid fixation, in the biomechanical study carried out by Meyer et al., neither material proved superior. There are also articles claiming that material-related complication rates reach $25 \%$ in triple arthrodesis done using rigid internal fixation ${ }^{[23]}$. In our cases, we used Kwires and did not encounter any problems.

The most common complication in triple arthrodesis is pseudoarthrosis. It is most commonly observed in talonavicular, calcaneocuboid and subtalar joints respectively 
[32, 33]. Pseudoarthrosis rates are reported in the literature as 133\%. ${ }^{[34,31,35,36]}$ Ahmed ${ }^{[27]}$ did not find pseudoarthrosis in any of a two-stage series of 7 cases. De Groot et al. ${ }^{[10]}$ reported a union rate of $57 \%$ in all joints in the series in which they applied only primary triple arthrodesis. This rate is given as $47 \%$ by Wülker, ${ }^{[36]}$ and $81 \%$ by Saltzman ${ }^{[34]}$. We did not observe any pseudoarthrosis in our series. While our results are similar to those obtained by Ahmed, ${ }^{[27]}$ who applied two stage arthrodesis, they are better than those of other studies carried out with triple arthrodesis alone. None of our patients developed delayed union. Insufficient cleaning of articular cartilages, inadequate contact of bone surfaces, lack or insufficiency of internal fixation, early loading and surgical mistakes are the main reasons for pseudoarthrosis ${ }^{[25,37,38]} \mathrm{We}$ associated our no-pseudoarthrosis findings with several factors: our patients were young and all non-smokers, there were no neurovascular problems, a stable fixation was applied, spongious parts of the bones removed from osteotomy lines were used as graft, and early loading was given. Avascular necrosis of the talus is an important complication in triple arthrodesis. It is considered to occur during talus resection to correct severe deformities, and to be due especially to damage of arterial structures at the talar dome ${ }^{[29]}$. AVN of the talus was found to be $6.5 \%$ by Duncan, and $9.9 \%{ }^{[29]}$ by Angus and Cowell, ${ }^{[25]}$ who carried out triple arthrodesis alone. In our series of 16 cases, we did not find any such complication.

Skin problems are also of importance in the surgical treatment of neglected clubfoot. Pell et al. [35] found that they encountered problems of superficial wounds in 4 of the 183 feet on which they applied triple arthrodesis without soft tissue release. El-Tayeby ${ }^{[26]}$ recorded that skin problems occurred in some soles of the 28 feet which were given trapezoid bone resection on cuboid with massive medial soft tissue release and that they were healed with wound care in a few days. Haddad et al. ${ }^{[39]}$ stated that among the 33 patients on which they performed revision triple arthrodesis, they observed superficial infection in 2, deep infection in 1, and wound opening in 1 other. In their study, Doðan et al. ${ }^{[31]}$ used triple arthrodesis and applied internal fixation on 12 of 30 feet, and they recorded that 2 developed superficial skin infection and 1 deep infection and skin necrosis, and that they grafted the necrosis. In the talectomy treatment of neglected pes equinovarus deformity on the 17 feet of 11 patients with neurovascular involvement, Yalçin et al. ${ }^{[40]}$ recorded superficial infection in the staple root in 4 feet, and in the incision area in 2. Infection location and incidence in our patients comply with the literature. We associate the fact that in patients for whom we added the extensile medial approach we did not observe more skin problems than those in the literature to good bleeding control, clean wound care and prophylactic antibiotic treatment. This study is limited by the lack of an adequate number of patients, long follow-up results and a comparison group, and the fact that the results were assessed by the same surgeon.

In conclusion, a single dorsolateral incision with meticulous soft tissue dissection and correct angle of wedging followed by strict postoperative care and protocol will definitely avoid both minor and major complications yielding a better cosmetically well accepted, pain free and functional plant grade foot. These efforts definitely changes the life of such patients.

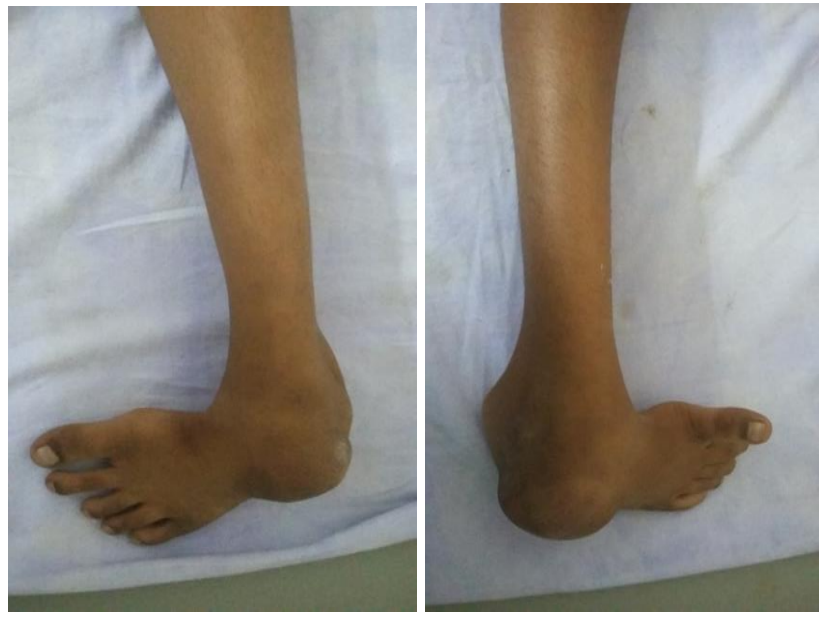

Preoperative clinical photo

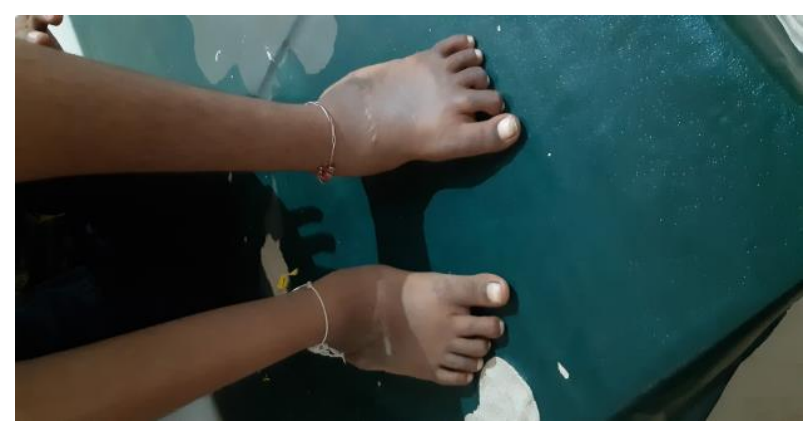

Post-operative clinical photo

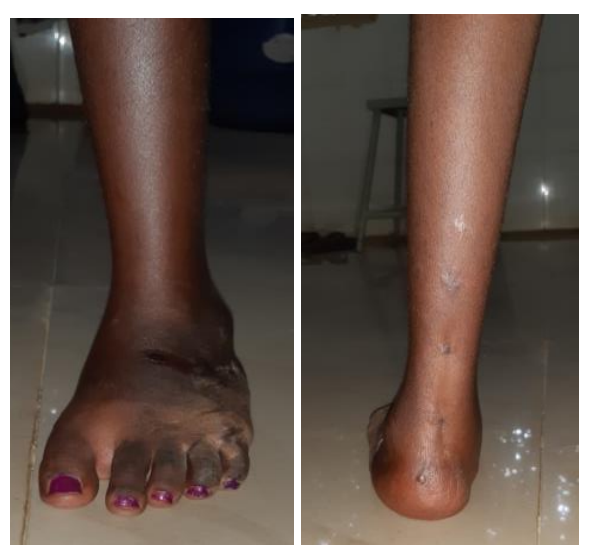

Percutaneous tendoachilis lengthening

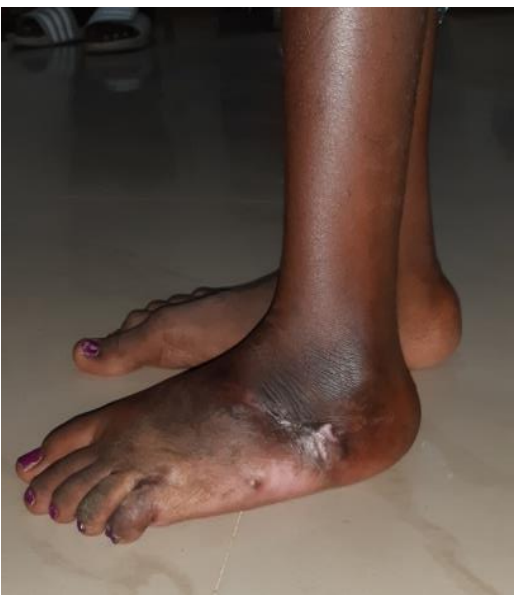

Single Dorso lateral incision 


\section{References}

1. Cummings RJ, Davidson RS, Armstrong $\mathrm{P}$ et al. Congenital clubfoot. AAOS instructional course lecture. J Bone Joint Surg [Am]. 2002; 84:290-308.

2. Davidson RS. Clubfoot salvage: a review of the past decade's contributions. J Pediatr Orthop. 2003; 23:410418.

3. Cummings RJ, Lovell WW. Operative treatment of congenital idiopathic club foot. J Bone Joint Surg Am. 1988; 70:1108-12.

4. Penny JN. The neglected clubfoot. Techniques in orthopaedics. 2005; 20:153-66.

5. Angus PD, Cowell HR. Triple arthrodesis. A critical long term review. J Bone Joint Surg [Br]. 1986; 68:260-265.

6. Ramseier LE, Schoeniger R, Vienne P, Espinosa N. Treatment of late recurring idiopathic clubfoot deformity in adults. Acta Orthop Belg. 2007; 73:641-7.

7. de Heus JA, Marti RK, Besselaar PP, Albers GH. The influence of subtalar and triple arthrodesis on the tibiotalar joint. A long-term follow-up study. J Bone Joint Surg. Br 1997; 79:644-7.

8. Vanderwilde R, Staheli LT, Chew DE, Malagon V. Measurements on radiographs of the foot in normal infants and children. J Bone Joint Surg Am. 1988; 70:407-15.

9. Mäenpää H, Lehto MU, Belt EA. What went wrong in triple arthrodesis? An analysis of failures in 21 patients. Clin Orthop Relat Res. 2001; 391:218-23.

10. de Groot IB, Reijman M, Luning HA, Verhaar JA. Longterm results after a triple arthrodesis of the hindfoot: function and satisfaction in 36 patients. Int Orthop 2008; 32:237-41.

11. Ponseti IV. Congenital Clubfoot: Fundamentals for Treatment. Oxford: Oxford University Press, 1996.

12. Pirani S, Zeznik L, Hodges D. Magnetic resonance imaging study of the congenital clubfoot treated with the Ponseti method. J Pediatr Orthop. 2001; 21:719-726.

13. Penny A. School access: children with motor disabilities in rural Uganda [MA Thesis Dissertation]. University of Victoria, 2001.

14. Yadav SS. Observations on operative management of neglected clubfoot. Int Orthop. 1981; 5:189-192.

15. Bitariho D, Penny JN. Triple arthrodesis in children for severe neglected clubfoot deformity. Presented at the Association of Surgeons of East Africa; Kampala, 2003.

16. Hersh A, Fuchs LA. Treatment of the uncorrected clubfoot by triple arthrodeses. Orthop Clin North Am. 1973; 4:103-115.

17. Herold HV, Torok G. Surgical correction of neglected club foot in the older child and adult. J Bone Joint Surg [Am]. 1973; 55:1385-1395.

18. Kitaoka HB, Alexander IJ, Adelaar RS, Nunley JA, Myerson MS, Sanders M. Clinical rating systems for the anklehindfoot, mid Foot, hallux, and lesser toes. Foot Ankle Int. 1994; 15:349-53.

19. Herold HZ, Torok G. Surgical correction of neglected club foot in the older child and adult. J Bone Joint Surg Am 1973; 55:1385-95.

20. Evans D. Relapsed club foot. J Bone Joint Surg. 1961; 43:722-33.

Abrams RC. Relapsed club foot. The early results of an evaluation of Dillwyn Evans' operation. J Bone Joint Surg Am. 1969; 51:270-82.

21. Lichtblau S. A medial and lateral release operation for club foot. A preliminary report. J Bone Joint Surg Am
$1973 ; 55: 1377-84$

22. Toohey JS, Campbell P. Distal calcaneal osteotomy in resistant talipes equinovarus. Clin Orthop Relat Res. 1985; 197:224-30.

23. Dwyer FC. The treatment of relapsed club foot by the insertion of a wedge into the calcaneus. J Bone Joint Surg. 1963; 45:67-75.

24. Hersh A, Fuchs LA. Treatment of the uncorrected clubfoot by triple arthrodesis. Orthop Clin North Am. 1973; 4:103-16.

25. Angus PD, Cowell HR. Triple arthrodesis. A critical longterm review. J Bone Joint Surg Br 1986; 68:260-5.

26. El-Tayeby HM. The neglected clubfoot: a salvage procedure. J Foot Ankle Surg 182 Acta Orthop Traumatol Turc. 1998; 37:501-9.

27. Ahmed AEO. Two stages of surgery for treatment of persistent or untreated congenital Talipes Equinovarus in young adult. Pan Arab J Orth Traum 1999; 3:51-7.

28. Dobbs MB, Nunley R, Schoenecker PL. Long-term follow- up of patients with clubfeet treated with extensive soft-tissue release. J Bone Joint Surg Am. 2006; 88:98696.

29. Duncan JW, Lovell WW. Hoke triple arthrodesis. J Bone Joint Surg Am 1978; 60:795-8.

30. Grill F, Franke J. The Ilizarov distractor for the correction of relapsed or neglected clubfoot. J Bone Joint Surg Br. 1987; 69:593-7.

31. Doğan A, Albayrak M, Uğur F, Zorer G. Triple arthrodesis in rigid foot deformities and the effect of internal fixation on clinical and radiographic results. [Article in Turkish] Acta Orthop Traumatol Turc. 2006; 40:220-7.

32. Van Dijk CN, Lim LS, Poortman A, Strübbe EH, Marti RK. Degenerative joint disease in female ballet dancers. Am J Sports Med. 1995; 23:295-300.

33. Chow S, Brandser E. Diagnostic and Therapeutic Foot and Ankle Injections. Semin Musculoskelet Radiol. 1998; 2:421-432.

34. Saltzman CL, Fehrle MJ, Cooper RR, Spencer EC, Ponseti IV. Triple arthrodesis: twenty-five and forty-fouryear average follow-up of the same patients. J Bone Joint Surg Am. 1999; 81:1391-402.

35. Pell RF, Myerson MS, Schon LC. Clinical outcome after primary triple arthrodesis. J Bone Joint Surg Am 2000; 82:47-57.

36. Wülker N, Flamme C. Hindfoot arthrodesis. [Article in German] Orthopade [Abstract]. 1996; 25:177-86.

37. Bednarz PA, Monroe MT, Manoli A. 2nd. Triple arthrodesis in adults using rigid internal fixation: an assessment of outcome. Foot Ankle Int. 1999; 20:356-63.

38. Graves SC, Mann RA, Graves KO. Triple arthrodesis in older adults. Results after long-term follow-up. J Bone Joint Surg Am. 1993; 75:355-62.

39. Haddad SL, Myerson MS, Pell RF. Schon LC. Clinical and radiographic outcome of revision surgery for failed triple arthrodesis. Foot Ankle Int. 1997; 18:489-99.

40. Yalçin S, Kocaoğlu B, Berker N, Erol B. Talectomy for the treatment of neglected pes equinovarus deformity in patients with neuromuscular involvement. [Article in Turkish] Acta Orthop Traumatol Turc. 2005; 39:316-21. 